\title{
Influence of Ageing with UV Radiation on Physicochemical Properties of Acrylic-Polyurethane Coatings
}

\author{
Danuta Kotnarowska \\ Kazimierz Pulaski University of Technology and Humanities, Radom, Poland \\ Email: d.kotnarowska@uthrad.pl
}

How to cite this paper: Kotnarowska, D. (2018) Influence of Ageing with UV Radiation on Physicochemical Properties of Acrylic-Polyurethane Coatings. Journal of Surface Engineered Materials and Advanced Technology, 8, 95-109.

https://doi.org/10.4236/jsemat.2018.84009

Received: July, 9, 2018

Accepted: September 23, 2018

Published: September 26, 2018

Copyright $\odot 2018$ by author and Scientific Research Publishing Inc. This work is licensed under the Creative Commons Attribution International License (CC BY 4.0).

http://creativecommons.org/licenses/by/4.0/

\section{cC) (i) Open Access}

\begin{abstract}
Investigated systems of acrylic-polyurethane coatings consisted of three kinds of coatings: base coat (acrylic), intermediate (polyurethane) and top coat (acrylic); each made up of two layers. Mean thickness of the coating system was equal $(145 \pm 1) \mu \mathrm{m}$. In time of accelerated UV ageing, electromagnetic radiation was emitted of wave length in the range (300 - 400) $\mathrm{nm}$ which was progressively inducing a destruction of coatings chemical structure, especially photooxidation and photolysis of coating material. Carried out X-ray investigations revealed increased contents of oxygen in aged coatings due to their photooxidation which resulted in increased tendency of coating microfragments to chip off from surface layer with the ageing time flow. The hardness increase of UV aged coatings was also observed which intensified their surface layer brittleness. This contributed to their thickness decrease by more than $10 \%$ after $2016 \mathrm{~h}$ of ageing. Photodestruction of UV aged coatings was documented as well by characteristics obtained during investigations carried out with DMA method use. A noticeable physical destruction of the coatings was observed in the form of silver cracks, etchings and grooves in surface layer as well as craters extending also into interlayer. Processes of physical and chemical destruction undergoing in aged coatings influenced roughness profile change and coatings surface topography shaping. After $2016 \mathrm{~h}$ of UV ageing, the roughness parameters $(\mathrm{Ra}, \mathrm{Rz}, \mathrm{Rt})$ increased several times. Long-lasting UV radiation influence on the surface of investigated acrylic-polyurethane coating systems contributed to their decorativeness loss. Microscopic examinations revealed colour change (yellowing) of blue pigment contained in polyurethane interlayer. Decline in coating gloss was also observed as the result of surface roughness increase.
\end{abstract}

\section{Keywords}

UV Radiation, Acrylic-Polyurethane Coatings 


\section{Introduction}

Polymer protective coatings of technical objects, in time of their operational life, are influenced by such ambient factors like solar radiation inducing photodestruction, aggressive media (acid rain, saline) or erosive particles (sand, gravel, hail) [1]-[8].

Among these factors, UV radiation is the dominating one causing polymer coatings ageing in the form of photooxidation being one of photodestruction kinds. The Sun is natural source of UV radiation emitting its different types divided into three bands: UV-A (wavelength 315 - $400 \mathrm{~nm}), \mathrm{UV}-\mathrm{B}(280-315 \mathrm{~nm})$ and UV-C (100 - $280 \mathrm{~nm})$. Radiation from the band UV-C and majority of radiation from the band UV-B is absorbed by the Earth's ozone layer and for this reason about $97 \%$ of ultraviolet radiation, which reaches the Earth's surface, belongs to the band UV-A. It causes photooxidation of polymer coatings undergoing primarily on their surface. Photooxidation is a radical process and presence of structural defects in polymer material promotes polymer chains disintegration (initiated by UV radiation) into free radicals, inducing sequential stages of polymer degradation [1] [2] [3] [4].

Chemical destruction of polymer structure under the influence of UV consists in its cross-linking increase or polymer chains breaking. It contributes to decrease of coating strength properties due to decline in coating material elasticity, impact strength and break strength value (in tensile test). In the case of cross-linking generated by UV radiation, mean particle mass increases as well as cross-linking density of polymer structure [9] [10]. Polymer coatings became than brittle and therefore susceptible to physical destruction (cracking) which conduces to fragments of polymer material chipping from the coating surface layer. The process of chipping leads to an increase of coating surface roughness parameters ( $\mathrm{Ra}, \mathrm{Rz}, \mathrm{Rt}$ ) [3] [4], which in consequence decreases gloss of the coating surface. Photodestruction redounds also to colour fading and local discolouration declining the coating decorativeness. As a result of humidity and impurities deposition in cavities of surface microroughness, the advantageous conditions occur for development of microorganisms, such as viruses, bacteria and mould fungus. They cause expansion of coating biological corrosion in the form of superficial pits and craters extending even to the substrate [1].

Moreover, so called silver cracks are generated under the influence of UV in superficial layers of coatings. They incorporate sets of pores of different dimensions-from nanometres to micrometers-which, in the reflected light of optical microscope, activate characteristic silver reflections validating their name [1].

Generation of nano- and micropores in coating material structure is the first stage of polymer coatings physical destruction under the influence of UV radiation. As a result of the pores coalescence with the time of ageing flow, capillaries develop, generating paths conducting aggressive media. The paths, in the final stage of their development, reach the substrate enabling transport of aggressive media to its surface which creates conditions favouring development of under- 
coating corrosion [4] [7]. The process of capillaries development speeds up macroscopic defects generation in coatings in the form of cracks, craters, macropores as well as fractures expansion between fillers and pigments surface and coating resin. Dimensions of conducting paths increase with the ageing time extension [8].

As the result of long lasting UV radiation action the photooxidation of polymer material occurs which leads to deterioration of polymer coatings physicochemical properties, such as mechanical strength, thermal resistance, resistance to aggressive media action, and interlayer adhesion as well as adhesion to the substrate. Thereby operational life of polymer coatings aged by UV deteriorates [2] [3] [4] [5].

Successful campaign against UV action negative effects requires recognition of polymer coatings destruction kinetics under the influence of UV radiation. For this purpose it is necessary to carry on investigations in special climatic chambers as investigation carried in natural conditions are very long-lasting (even 20 years). The results of such investigations could not be a base for life prediction of applied coatings because their formulas, for instance in automotive industry, change every five years on average.

Particular kind of polymer coating destruction under the influence of UV action depends on its chemical structure [1] [7] [8]. A proper choice of filler and pigments as well as coating forming material can decrease impact of UV influence on coatings destruction. For instance, aliphatic polymers are resistant to UV action as they are transparent to this kind of radiation and do not absorb it. Such property is presented by acrylates, polyvinyl acetate and aliphatic polyurethanes [11] [12].

As an efficient remedy, increasing the polymer coatings resistance to UV radiation, photostabilizers are added to their composition. The pigments, like carbon black or titanium dioxide, are special kind of these stabilizers [13]. Increase of the coatings resistance to the impact of UV radiation can be also obtained in a result of their modification with fillers, especially nanofillers made of silica, aluminium trioxide or zinc monoxide [11] [14]-[26].

\section{Experimental Details}

\subsection{Materials and Samples Preparation}

Coating systems, commonly used in car body repair workshops, were investigated. The samples of coating systems were applied on the substrate which was surface of steel plates, of dimensions $170 \times 90 \times 1.5 \mathrm{~mm}$, made of constructional killed steel S 235 JRG 2 (acc. PN-EN 10025-1:2007). Before primer layer application, surface of each plate was firstly cleaned with abrasive paper of grade P80 and then degreased with extraction solvent.

The samples of coating systems were obtained by air spraying onto steel plates surface of successive three kinds of polymeric coatings, which were as follows: two-layer primer, two-layer intermediate coating-interlayer, tinting the coating 
system blue, and two-layered top coat. Two layers of primer were obtained from solvent based acrylic paint containing zinc pigment. After primer drying, its surface was wet grinded with abrasive paper of grade 600 . In the next stage the grinded primer surface was degreased with extraction solvent. As the consecutive air-sprayed coating, the interlayer was applied made of waterborne polyurethane paint which contained grained pigments: aluminium trioxide, iron oxide and carbon black. Three-layer top coat was made of colourless HS acrylic lacquer. Obtained coating systems were acclimatized for 20 days at temperature $20^{\circ} \mathrm{C} \pm 2{ }^{\circ} \mathrm{C}$ (acc. PN-EN 23270:1993).

\section{Conditions of coatings ageing with UV radiation}

Ageing of coating systems under the influence of UV radiation was carried out using two lamps LRF 250 E40 without glass casing, of power $250 \mathrm{~W}$ each. These lamps emitted electromagnetic radiation of wave length in the range 300 $460 \mathrm{~nm}$ onto coating samples situated in the distance of $300 \mathrm{~mm}$. Maximum ageing period was equal $2016 \mathrm{~h}$ (84 days).

\subsection{Methodics of Coatings Physico-Chemical Properties Examining}

Before investigation of acrylic-polyurethane coating systems ageing, the examinations of their thickness and dynamic strength were carried out as well as examinations of their surface hardness, roughness, gloss, and morphology.

These examinations were repeated after each finished cycle of ageing investigation that is after $336 \mathrm{~h}, 672 \mathrm{~h}, 1080 \mathrm{~h}, 1344 \mathrm{~h}, 1680 \mathrm{~h}$ and $2016 \mathrm{~h}$.

\section{Methodics of coating thickness examination}

Thickness of acrylic-polyurethane coating systems was examined (acc. PN-EN ISO 2808:2008) using coating thickness meter Mega-Check FE made by List-Magnetik GmbH.

The mean thickness of investigated coating systems was equal (145 \pm 1$) \mu \mathrm{m}$, including thickness of the primer coat $(50 \pm 1) \mu \mathrm{m}$, intermediate coat $(35 \pm 1)$ $\mu \mathrm{m}$ and top coat $(60 \pm 1) \mu \mathrm{m}$. Thickness of the coatings were measured after their drying up.

Methodics of coating hardness examination

Hardness of acrylic top coats was evaluated using the method of pencil hardness test (acc. PN-EN ISO 15184:2013-04).

Methodics of coating surface roughness and topography examination

Topography of coating system surface as well as its roughness were analysed on the basis of the characteristics obtained with the help of Taylor Hobson interferometric microscope Talysurf CCI. It operates on the principle of white light interferometry enabling optical measurements of roughness, waviness and coating surface topography. Evaluation of coating surface roughness was carried out according to PN-M-04251:1987 and PN-EN ISO 8501-1:2008 requirements.

Methodics of coating surface morphology examination

Physical destruction of aged acrylic top coat surface was evaluated using field emission SEM Hitachi SU-70, equipped with energy dispersive X-ray spectrometer 
(EDS) made by Thermo Scientific, giving possibility for qualitative evaluation of chemical composition of examined coatings superficial layers (up to $1 \mu \mathrm{m}$ in depth).

Surface destruction of coatings aged with UV was evaluated also on the grounds of surface pictures obtained with the use of optical microscope Studar Lab Met made by PZO (Polish Optical Works).

Methodics of coating surface gloss examination

Mirror gloss of acrylic top coats was measured using Elcometer gloss meter NOVO-GLOSS, for light beam incidence angle values $(20,60 \text {, and } 85)^{\circ}$ (acc. PN-EN ISO 2813:2014-11).

\section{Methodics of coating dynamic mechanical analysis (DMA)}

Dynamical strength (rheological properties) of acrylic-polyurethane coating systems was examined using Polymer Laboratories equipment PL-DMTA (Dynamic Mechanical Thermal Analyser) MkII. The examinations consist in subjecting coating samples in the form of foil strap to sinusoidal cycles of stretching at frequency $1 \mathrm{~Hz}$ and deformation amplitude $16 \mu \mathrm{m}$ after prior static stretching at tension equal 2.5 - 3.0 MPa. Coating samples were examined under the influence of increasing temperature in the range $(0-200)^{\circ} \mathrm{C}$, at heating speed $3^{\circ} \mathrm{C} / \mathrm{min}$.

\section{Results}

Surface photooxidation of coatings aged with UV radiation are documented by spectrographs obtained using energy dispersive X-ray spectrometer (EDS) cooperating with SEM Hitachi SU-70. The spectrographs presented in Figure 1 show increase of oxygen contents in examined aged superficial layer (of $1 \mu \mathrm{m}$ thickness) with the flow of time.

Dynamic mechanical thermal analysis, carried out using Polymer Laboratories analyzer MkII, revealed essential influence of ageing changes, occurred in coatings as a result of UV action, on the following characteristics: dynamic loss fac$\operatorname{tor}(\operatorname{tg} \delta$ ), the dynamic storage modulus (E'), and elongation (L) (Figures 2-4).

$\operatorname{Tg} \delta$ characteristics in the function of temperature within the range (0 $150)^{\circ} \mathrm{C}$ shows decline in peak of curve representing UV aged coating, comparing with curve representing unaged coating (Figure 2). This confirms development of photodestruction processes in the coating structure which causes slight increase of glass transition temperature $\mathrm{Tg}$ (corresponding to the peak of the curve $\operatorname{tg} \delta$ ) from the value $76.77^{\circ} \mathrm{C}$ (for the unaged coating) to the value $77.29^{\circ} \mathrm{C}$ (for the coating aged with UV for $2016 \mathrm{~h}$ ). This can testify to polymer material additional crosslinking [16] leading to stress generation in aged coating structure. Thereby coatings tendency to cracking increases with the ageing period increase. On account of cracks development in coating structure, the tendency to coating fragments chipping from the surface layer increases.

Physical and chemical destruction of acrylic-polyurethane coatings caused decrease of their dynamic storage modulus $\left(E^{\prime}\right)$ in temperature range $(0-65)^{\circ} \mathrm{C}$, testifying elastic properties deterioration (Figure 3 ). This has influence on coating susceptibility to physical photodestruction in the form of furrows. 


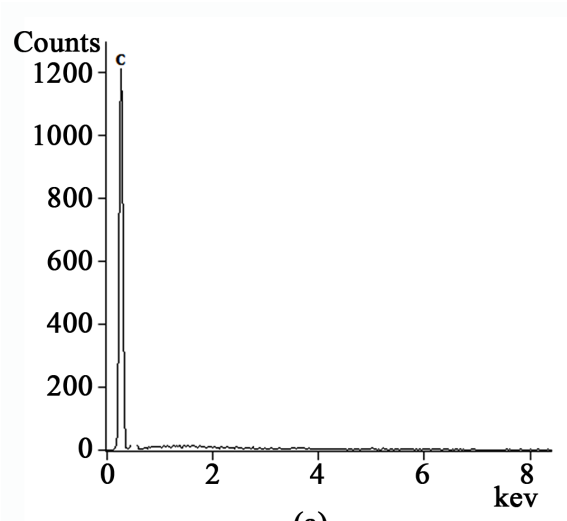

(a)

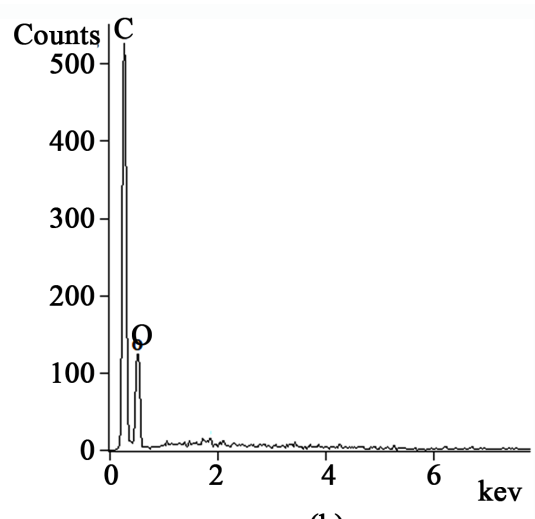

(b)

Figure 1. Spectrograph obtained from surface of acrylic-polyurethane coating aged with $\mathrm{UV}$ radiation for $0 \mathrm{~h}(\mathrm{a})$ and $2016 \mathrm{~h}(\mathrm{~b})$.

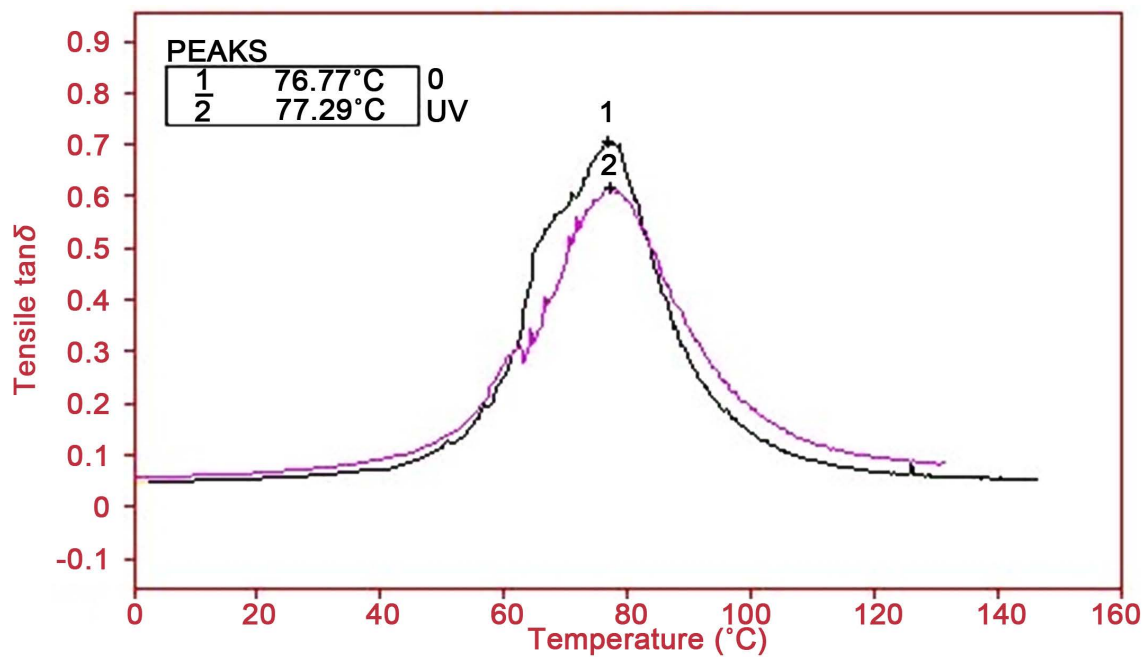

Figure 2. Dynamic loss factor $(\operatorname{tg} \delta$ ) in temperature function of acrylic-polyurethane coatings unaged (1) and aged with UV radiation for $2016 \mathrm{~h}$ (2).

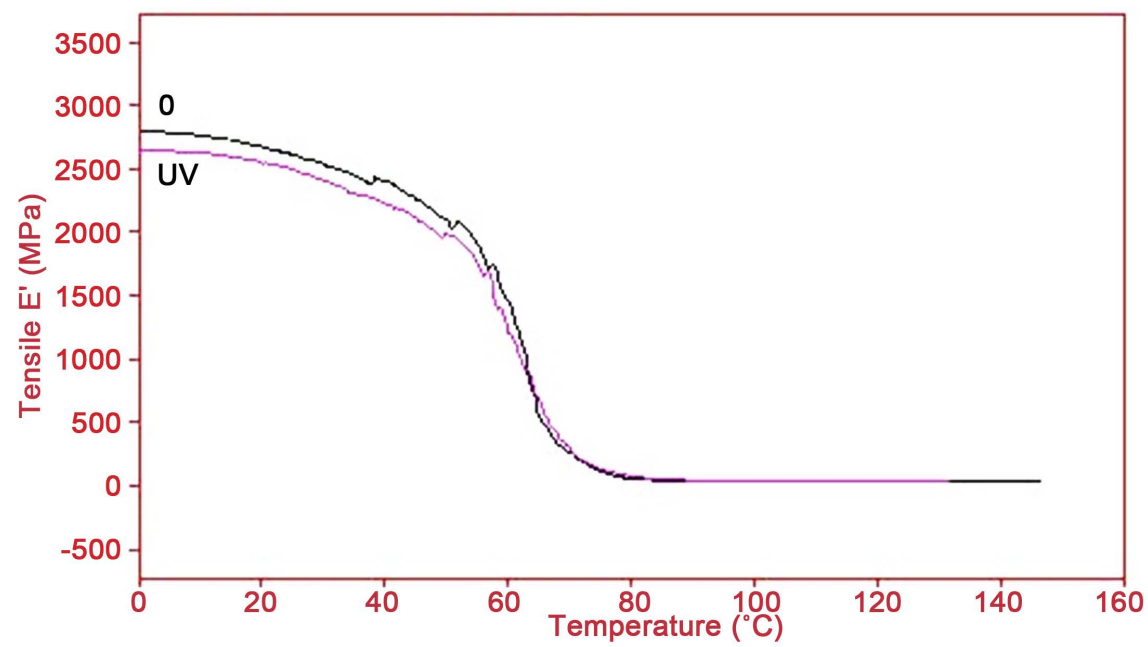

Figure 3. Dynamic storage modulus E' in temperature function of acrylic-polyurethane coatings unaged (0) and aged with UV radiation for $2016 \mathrm{~h}$ (UV). 
Moreover, elongation decrease (by 12\%) of UV aged coatings was observed as well as the value of break temperature decrease by more than 13\% (Figure 4). Such phenomenon was caused by an increase of coatings ageing degree under the influence of UV radiation resulting from their oxidation increase (Figure 1) which was documented by the increase of carbonyl groups content [3] [4]. Aged coatings revealed also bigger porosity. Degradation and destruction of the coatings under the UV influence induced, among others, coatings brittleness increase resulting in their elasticity decline (Figure 3) which in turn resulted in the elongation decrease (Figure 4) observed during elongation test [1] [3] [4].

Long-lasting UV radiation influence on surface of examined acrylic coatings contributed essentially to their gloss decrease. Examination made using gloss meter revealed decrease of coating surface gloss, for example, for light incidence angle $\alpha=20^{\circ}$ by $98 \%$, for $\alpha=60^{\circ}$ by $86 \%$, and for $\alpha=85^{\circ}$ by $62 \%$ (Figure 5 ).

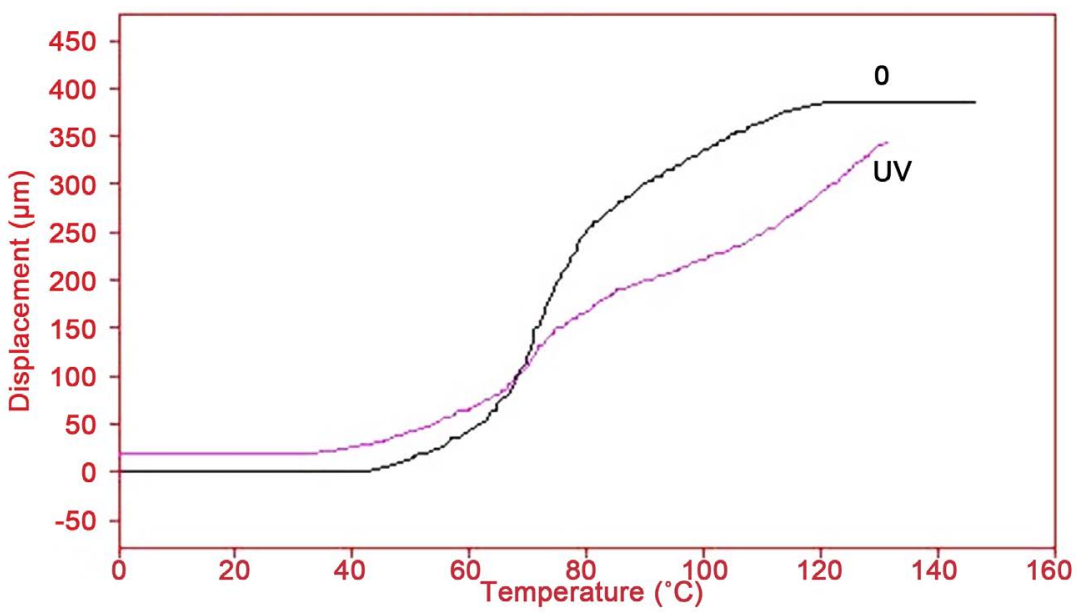

Figure 4. Elongation (L) in temperature function of acrylic-polyurethane coatings unaged (0) and aged with UV radiation for $2016 \mathrm{~h}$ (UV).

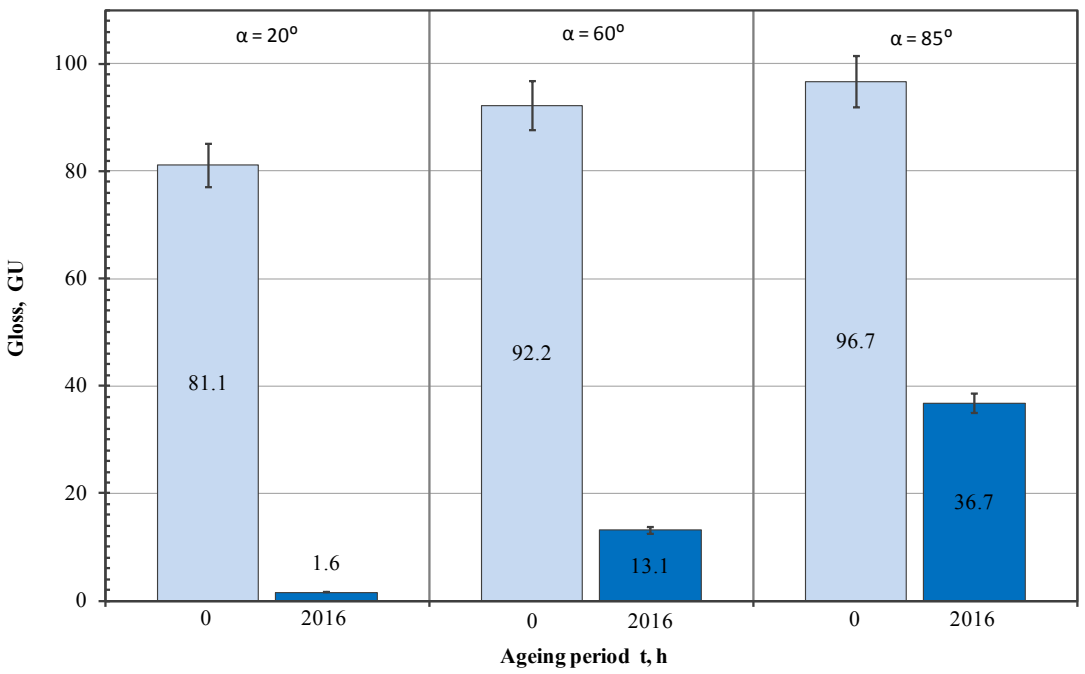

Figure 5. Influence of UV ageing for $2016 \mathrm{~h}$ on the gloss of acrylic top coat, for light incidence angle $\alpha=(20,60 \text { and } 85)^{\circ}$. 
The reason of aged coating discoloration was colour change of blue pigment contained in the interlayer which was proved by yellowing of pigments in polyurethane coating (Figure 6), as it was stated during microscopic observation.

Coating systems UV ageing caused also increase of their surface layer pencil hardness from $\mathrm{H}$ value before ageing to $6 \mathrm{H}$ value after ageing for $2016 \mathrm{~h}$. Hardness increase induced brittleness of acrylic top coat increase what brought about its surface roughness increase. Moreover, an increased susceptibility of the coatings to brittle cracking accompanied their hardness increase [2] [4].

Carried out investigations of acrylic top coat surface state proved that with UV ageing period extension roughness parameters increased several times. They increased as follows: Ra 6 times, Rz more than 5 times, and Rt more than 4 times (Table 1 and Table 2, Figure 7 and Figure 8). It was caused by microfragments flaking from surface layers as the result of their brittleness increase. Additional cross-linking and photooxidation of surface layer as well as silver cracking development in it was the reason of this destruction type.

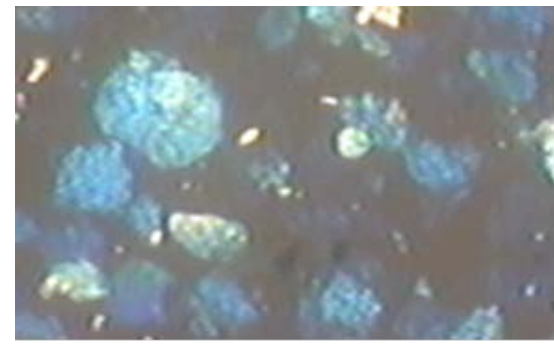

(a)

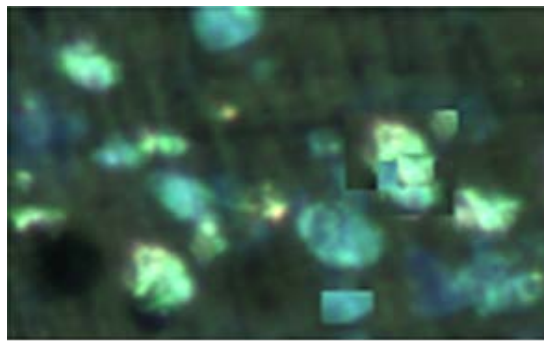

(b)

Figure 6. Morphology of blue acrylic-polyurethane coating (interlayer) unaged (a) and aged with UV radiation for $2016 \mathrm{~h}$; (b) visible through transparent acrylic top coat (magnification $100 \times$ ).

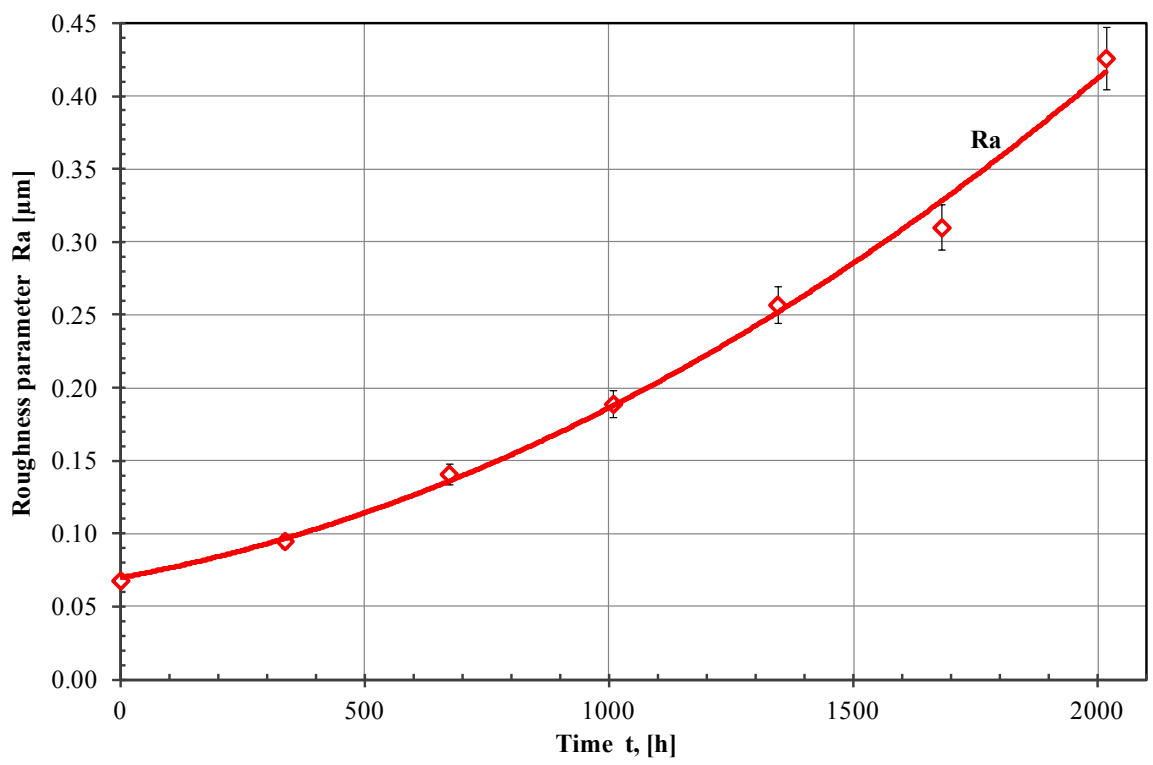

Figure 7. Influence of UV ageing on the roughness parameter Ra of acrylic-polyurethane coating systems. 
Table 1. Surface roughness profiles of acrylic-polyurethane coating systems aged with UV radiation for $(0-2016) \mathrm{h}$.

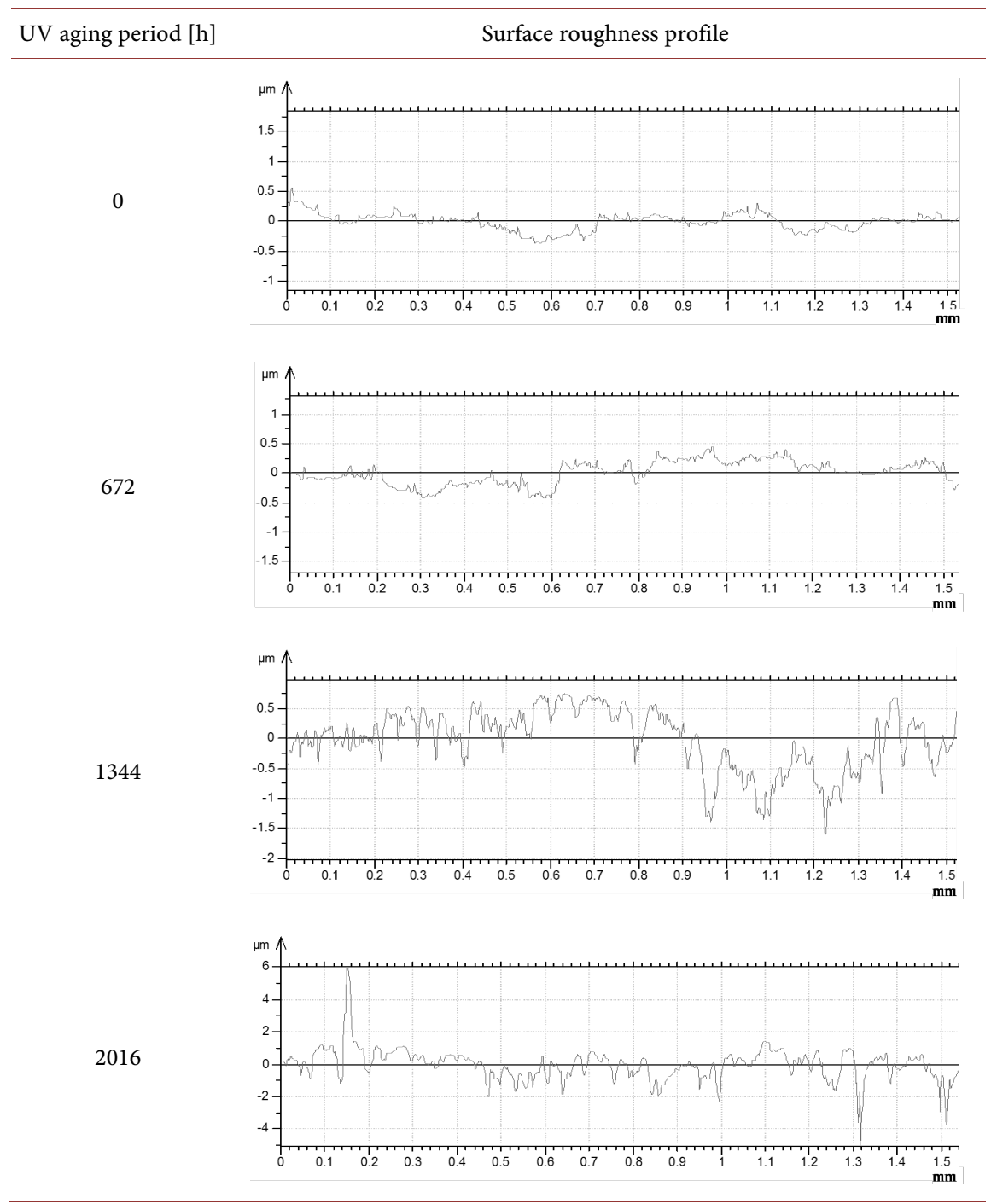

Table 2. Mathematical models describing trend curves for roughness parameters (presented in Figure 7 and Figure 8) of acrylic-polyurethane coatings systems.

\begin{tabular}{cccc}
\hline No. & $\begin{array}{c}\text { Parameter of } \\
\text { surface roughness }\end{array}$ & Equation of trend line & $\begin{array}{c}\text { Coefficient of } \\
\text { determination } \mathrm{R}^{2}\end{array}$ \\
\hline 1 & $\mathrm{Ra}$ & $\mathrm{y}=5 \mathrm{E}-08 \mathrm{x}^{2}+6 \mathrm{E}-05 \mathrm{x}+0.069$ & 0.995 \\
2 & $\mathrm{Rz}$ & $\mathrm{y}=1 \mathrm{E}-06 \mathrm{x}^{2}+1.261$ & 0.991 \\
3 & $\mathrm{Rt}$ & $\mathrm{y}=4 \mathrm{E}-07 \mathrm{x}^{2}+0.380$ & 0.983 \\
\hline
\end{tabular}

UV radiation influence on examined acrylic-polyurethane coatings systems contributed to their physical destruction, what was proved by the results of surface topography investigations presented in Table 3. Pictures of surface topography obtained for ageing longer than $1008 \mathrm{~h}$ revealed presence of parallel grooves, so called silver cracks, characteristic for polymer surfaces subjected to UV action. 
Table 3. Surface topography of acrylic-polyurethane coating system aged with UV radiation.
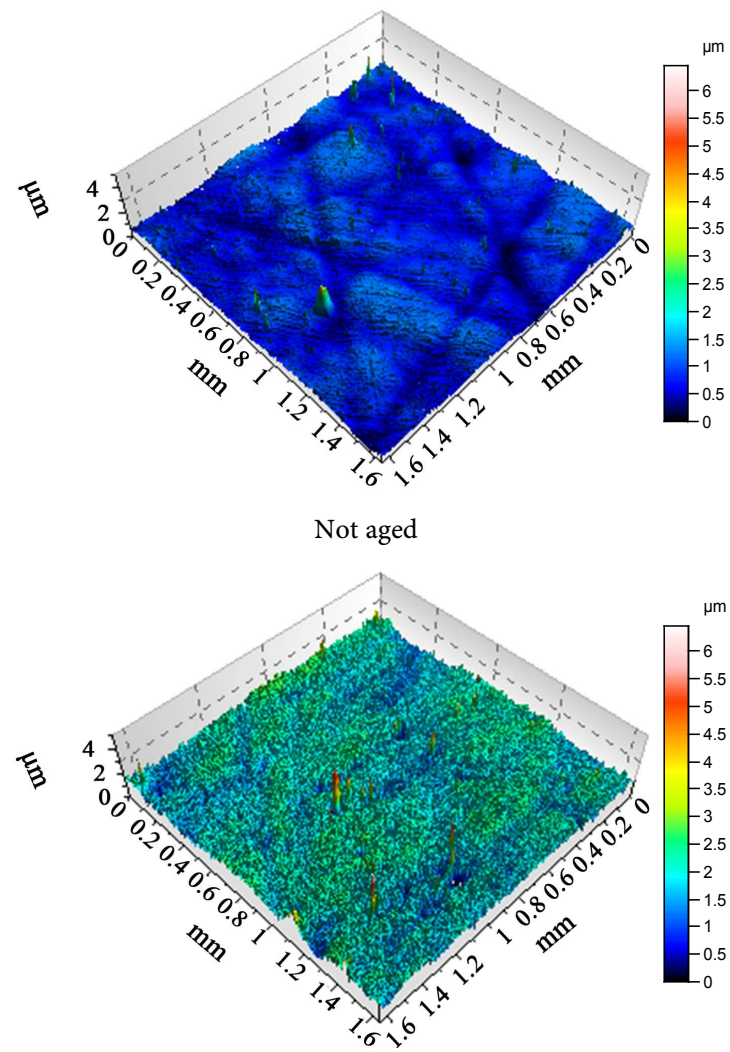

Aged for $1680 \mathrm{~h}$

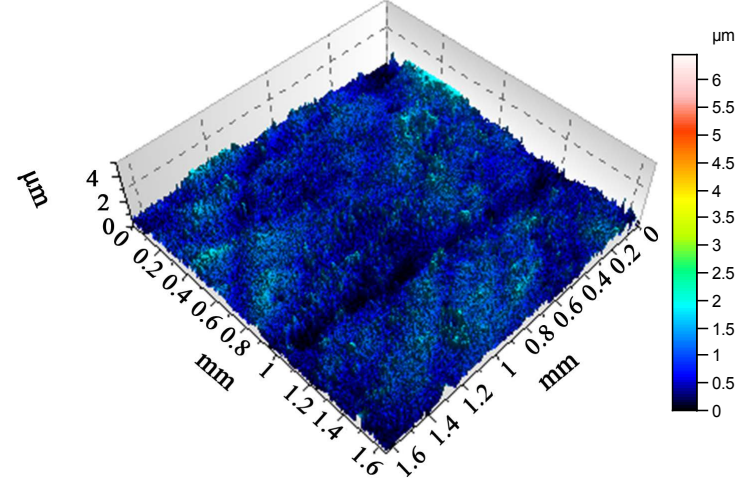

Aged for $1008 \mathrm{~h}$

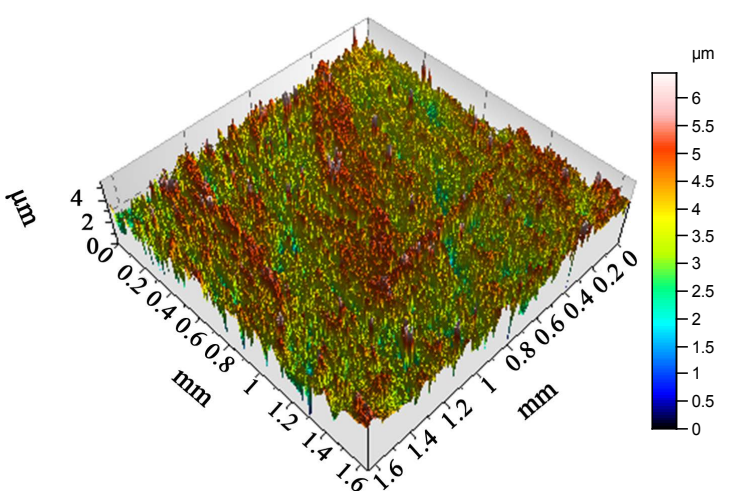

Aged for $2016 \mathrm{~h}$

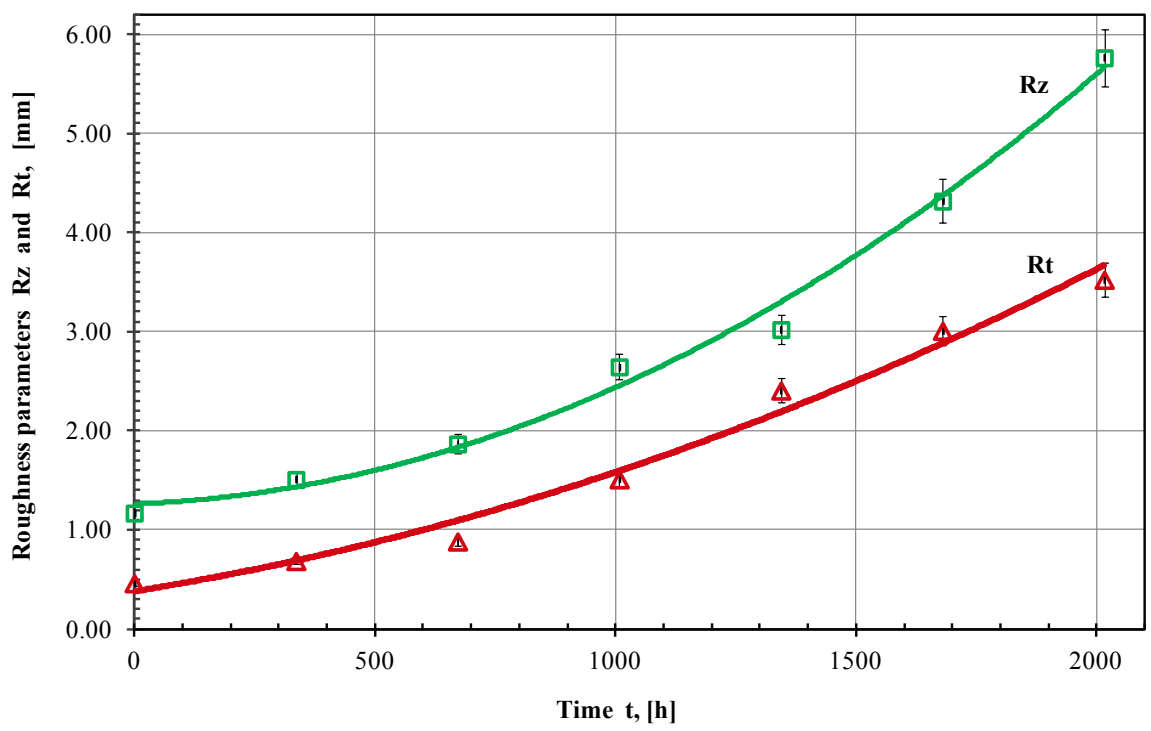

Figure 8. Influence of UV ageing on the roughness parameters Rz and Rt of acrylic-polyurethane coating systems.

Existence of characteristic grooves in coatings aged with UV radiation was also attested by the results of surface morphology examination carried out with SEM use (Table 4). Presence of silver cracks on coating surface was detected 
Table 4. Morphology of acrylic-polyurethane coatings aged with UV radiation.

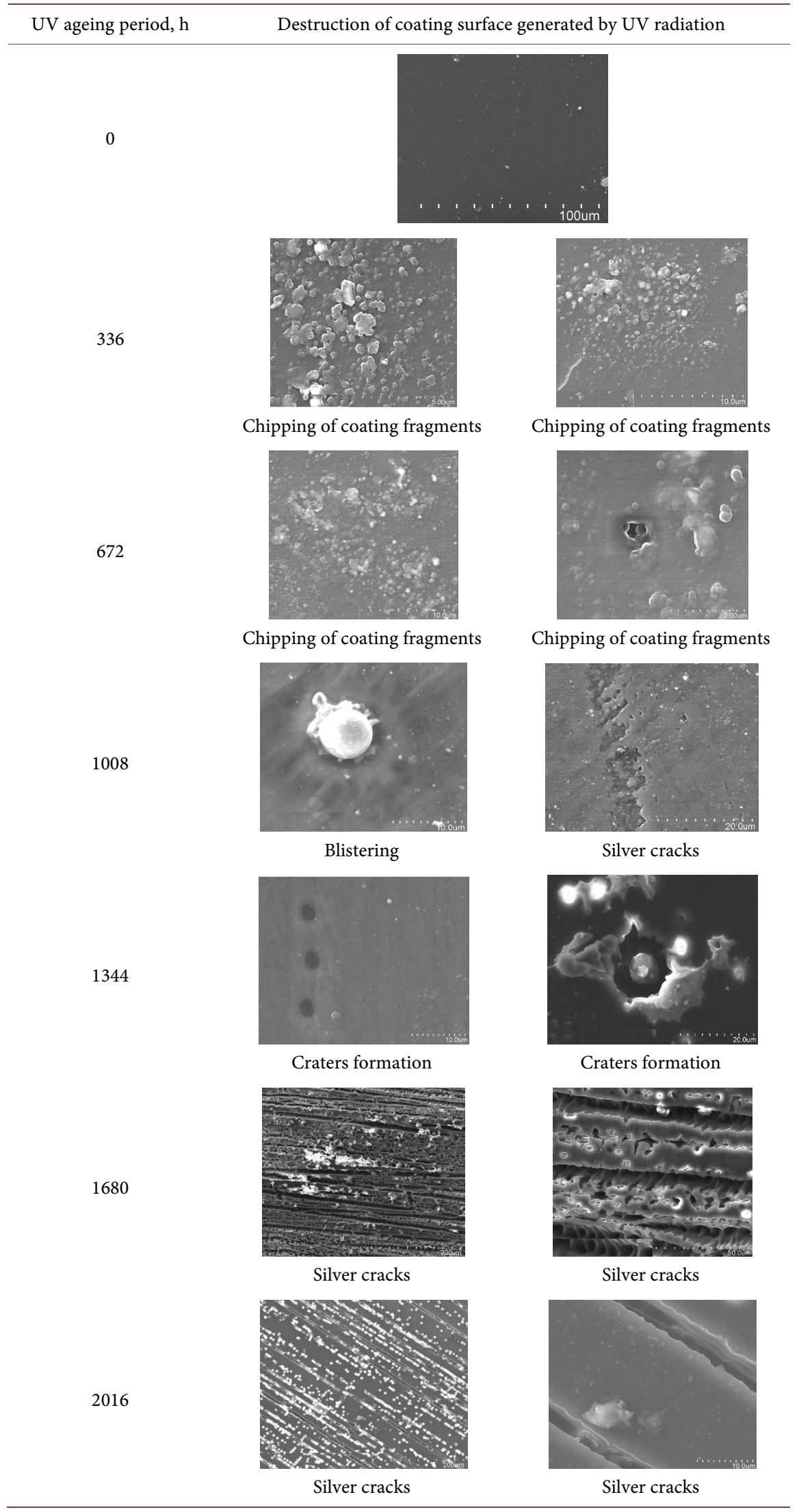


already after 1080 hours of UV ageing. Dimensions of superficial silver cracks, developing in acrylic top coat, increased with the ageing time flow. Moreover, craters and etchings appeared on the surface of aged coatings. The craters were appearing during final phase of ageing as a result of pigments and fillers chipping from interlayer which reason should be seen in adhesion loss of pigments and fillers to the coating forming resin on account of it photodestruction.

\section{Summary}

Carried out investigations showed that UV ageing induces destruction of chemical structure of acrylic-polyurethane coating systems. It is revealed by carbonyl groups content increase which testifies the intensification of coatings oxidation. Processes of photooxidation are initiated under the influence of UV radiation in superficial coatings, which was verified using X-ray spectrometry. Obtained spectra revealed increased contents of oxygen in aged coatings which resulted in increased tendency of coating microfragments to chip off from surface layer with the ageing time flow. Heavy physical destruction of coatings was observed in the form of etchings and grooves in surface layer as well as craters spanning also interlayer.

Characteristics obtained during investigations carried out with DMA method use disclosed photodestruction of coatings aged with UV radiation. In particular, this type of destruction was evidenced by the dynamic loss factor $(\operatorname{tg} \delta)$ characteristics as they revealed a peak decline of $\operatorname{tg} \delta$ in temperature function curve. What more, glass transition temperature increase was observed what might testify additional cross-linking of coatings. Physical and chemical destruction of acrylic-polyurethane coatings resulted in decrease of the dynamic storage modulus E' (in temperature range $0^{\circ} \mathrm{C}-65^{\circ} \mathrm{C}$ ), which proved decline of coating elastic properties.

Moreover, in the case of coatings aged with UV radiation, decrease of their elongation was observed in elongation test as the result of brittleness increase. Such phenomenon is caused by escalation of coatings ageing degree under the influence of UV radiation as a result of their oxidation development evidenced by the elevated content of carbonyl groups. Aged coatings show also increased porosity. DMA investigations revealed also the temperature decline of coatings break. Therefore, the thermal resistance of aged coatings decreased too.

The process of chipping increase with ageing time flow has the essential influence on significant growth of surface roughness profile and change of aged coatings surface topography.

Development of ageing processes in surface layer caused hardness of coatings increase escalating also their brittleness. Surface layer brittleness of coatings aged with UV contributed to their thickness decrease by more than $10 \%$ after $2016 \mathrm{~h}$ of ageing.

Coatings ageing with UV radiation caused generation of characteristic parallel grooves visible on topography pictures of coatings aged longer than $1000 \mathrm{~h}$. These grooves constituted zones of silver cracks, which was proved by results of 
aged coatings morphology investigations with SEM use.

Presence of silver cracks on coatings surface was stated already after UV ageing for $1008 \mathrm{~h}$. The cracks developed from the coatings surface to their inside. Length, width and depth of silver cracks increased with ageing time flow. Moreover, sparse etchings and craters reaching inside of the interlayer occurred in the aged coatings. The etchings in acrylic top coat arose already in initial period of UV ageing (after $600 \mathrm{~h}$ ), whereas the craters appeared only in final ageing period (after $2016 \mathrm{~h}$ ). The craters development was caused by local chipping of pigments and fillers from interlayer as they lost adhesion to the coating resin in the result of its photodestruction.

Coating systems ageing with UV radiation also caused increase of their surface layer pencil hardness from $\mathrm{H}$ value before ageing to $6 \mathrm{H}$ value after ageing for 2016 h. Hardness increase induced brittleness of acrylic top coat increase what brought about its surface roughness increase. What more, with coatings hardness increase their tendency to brittle cracks generation elevated too [2] [4].

Processes of physical and chemical destruction undergoing in aged coatings influenced roughness profile change and coatings surface topography shaping. Increase of coating surface roughness parameters was observed with UV ageing period increase. Its principal reason lies in pigment and filler grains chipping from top coats structure as a result of adhesion loss between their surface and polymer material. The top coats components tendency to chipping raises with oxidation degree increase of polymer material elevating with elongation of UV ageing period. After $2016 \mathrm{~h}$ of UV ageing, the roughness parameters increased several times. It was caused by microfragments chipping from aged coatings surface followed by brittleness increase of surface coatings due to chemical structure of coating resin photodestruction, as well as silver cracks development.

Long-lasting UV radiation influence on surface of investigated acrylic-polyurethane coating systems contributed to their decorativeness loss. Microscopic examinations revealed colour change of blue pigment contained in polyurethane interlayer. The pigment has changed colour already after $2016 \mathrm{~h}$ of UV ageing causing coatings fading. Deterioration of coating gloss was also observed as the result of surface roughness increase.

\section{Conflicts of Interest}

The author declares no conflicts of interest regarding the publication of this paper.

\section{References}

[1] Kotnarowska, D. (2013) Destruction of Polymer Coatings under the Operational Factors Influence. In: Monograph, Ed., Kazimierz Pulaski University of Technology and Humanities, Radom.

[2] Kotnarowska, D. (2010) Epoxy Coating Destruction as a Result of Sulphuric Acid Aqueous Solution Action. Progress in Organic Coatings, 67, 324-328. https://doi.org/10.1016/j.porgcoat.2009.10.026

[3] Kotnarowska, D. (1999) Influence of Ultraviolet Radiation and Aggressive Media on 
Epoxy Coating Degradation. Progress in Organic Coatings, 37, 149-159. https://doi.org/10.1016/S0300-9440(99)00070-3

[4] Kotnarowska, D. (2006) Influence of Ultraviolet Radiation on Erosive Resistance of Modified Epoxy Coatings. Solid State Phenomena, 113, 585-588.

https://doi.org/10.4028/www.scientific.net/SSP.113.583

[5] Kotnarowska, D. (2006) Examination of Dynamics of Polymeric Coatings Erosive Wear Process. Materials Science-Medźiagotyra, 12, 138-143.

[6] Nguyen, T., Hubbard, J.B. and Pommersheim, J.M. (1996) Unified Model for the Degradation of Organic Coatings on Steel in a Neutral Electrolyte. Journal of Coatings Technology, 855, 45-56.

[7] Perera, D.Y. (1994) Physical Ageing of Organic Coatings. Progress in Organic Coatings, 47, 61-76. https://doi.org/10.1016/S0300-9440(03)00037-7

[8] Pommersheim, J.M., Nguyen, T., Zhang, Z. and Hubbard, J.B. (1994) Degradation of Organic Coatings on Steel. Progress in Organic Coatings, 25, 23-41. https://doi.org/10.1016/0300-9440(94)00501-X

[9] Bauer, D.R., Dickie, R.A. and Koenig, J.L. (1986) Cure and Photodegradation of Two-Package Acrylic/Urethane Coatings. Industrial and Engineering Chemistry. Product Research and Development, 25, 289-296. https://doi.org/10.1021/i300022a028

[10] Bauer, D.R., Gerlock, J.L., Mielewski, D.F., Paputa, Peck, M.C. and Carter III, R.O. (1991) Photodegradation and Photostabilization of Urethane Crosslinked Coatings. Industry Engineering and Chemical Research, 30, 2482-2487. https://doi.org/10.1021/ie00059a019

[11] Kotlík, P., Doubravová, K., Horálek, K.J., Kubáč, L. and Akrma, J. (2014) Acrylic Copolymer Coatings for Protection against UV Rays. Journal of Cultural Heritage, 15, 44-48. https://doi.org/10.1016/j.culher.2013.01.002

[12] Pintus, V., Wei, S. and Schreiner, M. (2014) Accelerated UV Ageing Studies of Acrylic, Alkyd, and Polyvinyl Acetate Paints Influence of Inorganic Pigments. Microchemical Journal, 124, 949-961. https://doi.org/10.1016/j.microc.2015.07.009

[13] Saha, S., Kocaefe, D., Boluk, Y. and Pichette, A. (2011) Enhancing Exterior Durability of Jack Pine by Photo-Stabilization of Acrylic Polyurethane Coating Using Bark Extract. Part 1: Effect of UV on Colour Change and ATR-FT-IR Analysis. Progress in Organic Coatings, 70, 376-382. https://doi.org/10.1016/j.porgcoat.2010.09.034

[14] Barna, E., Bommer, B., Kürsteiner, J., Vital, A., Trzebiatowski, O., Koch, W., Schmid, B. and Graule, T. (2005) Innovative, Scratch Proof Nanocomposites for Clear Coatings. Composites: Part A, 36, 473-480. https://doi.org/10.1016/j.compositesa.2004.10.014

[15] Bauer, F., Flyunt, R., Czihal, K., Langguth, H., Mehnert, R., Schubert, R. and Buchmeiser, M.R. (2007) UV Curing and Matting of Acrylate Coatings Reinforced by Nano-Silica and Micro-Corundum Particles. Progress in Organic Coatings, 60, 121-126. https://doi.org/10.1016/j.porgcoat.2007.07.005

[16] Bauer, F., Decker, U., Czihal, K., Mehnert, R., Riedel, C., Riemschneider, M., Schubert, R. and Buchmeiser, M.R. (2005) UV Curing and Matting of Acrylate Nanocomposite Coatings by $172 \mathrm{~nm}$ Excimer Irradiation. Progress in Organic Coatings, 64, 474-481. https://doi.org/10.1016/j.porgcoat.2008.08.009

[17] Bauer, F., Decker, U., Naumov, S. and Riedel, C. (2014) Photoinitiator-Free UV Curing and Matting of Acrylate-Based Nanocomposite Coatings (Part 3). Progress 
in Organic Coatings, 77, 1085-1094. https://doi.org/10.1016/j.porgcoat.2014.03.013

[18] Graule, T. (2005) Innovative, Scratch Proof Nanocomposites for Clear Coatings. Composites (Part A), 36, 473-480.

https://doi.org/10.1016/j.compositesa.2004.10.014

[19] Dickie, R.A. (1994) Paint Adhesion, Corrosion Protection and Interfacial Chemistry. Progress in Organic Coatings, 25, 3-22. https://doi.org/10.1016/0300-9440(94)00500-1

[20] Kotnarowska, D. (2009) Effect of Nanofillers on Wear Resistance of Polymer Coatings. Solid State Phenomena, 144, 285-290. https://doi.org/10.4028/www.scientific.net/SSP.144.285

[21] Kotnarowska, D. (2008) Influence of Mechanical Factors on Surface State of Acrylic Coatings with Nanofillers. Materials Science-Medźiagotyra, 14, 337-340.

[22] Kotnarowska, D., Przerwa, M. and Wojtyniak, M. (2014) Influence of Polymer Coatings Modification with Nanoparticles on Their Erosion. Journal of Vibroengineering, 13, 870-876

[23] Unsworth, J. and Li, Y. (1992) Thermal Degradation of Epoxy/Silica Composites Monitored via Dynamic Mechanical Thermal Analysis. Journal of Applied Polymer Science, 46, 1375-1379. https://doi.org/10.1002/app.1992.070460806

[24] Zhai, Z., Li, G., Liu, Z. and Chang, X. (2016) The Anti-Ultraviolet Light (UV) Aging Property of Aluminium Particles/Epoxy Composite. Progress in Organic Coatings, 101, 305-308. https://doi.org/10.1016/j.porgcoat.2016.09.006

[25] Asmatulu, R., Mahmud, G.A., Hille, C. and Misak, H.E. (2011) Effects of UV Degradation on Surface Hydrophobicity, Crack, and Thickness of MWCNT-Based Nanocomposite Coatings. Progress in Organic Coatings, 72, 553-561. https://doi.org/10.1016/j.porgcoat.2011.06.015

[26] Bondioli, F., Cannillo, V., Fabbri, E. and Messori, M. (2006) Preparation and Characterization of Epoxy Resins Filled with Submicron Spherical Zirconia Particles. Polimery, 5, 789-794. 